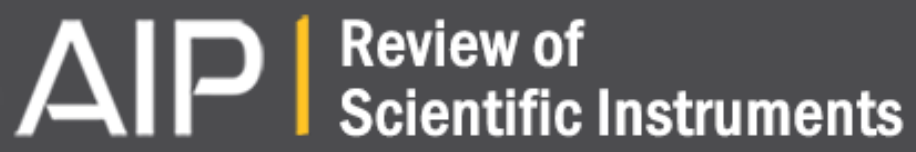

\section{Imaging photomultiplier array with integrated amplifiers and high-speed USB interfacea)}

M. Blacksell, J. Wach, D. Anderson, J. Howard, S. M. Collis, B. D. Blackwell, D. Andruczyk, and B. W. James

Citation: Review of Scientific Instruments 79, $10 \mathrm{F506}$ (2008); doi: 10.1063/1.2965013

View online: http://dx.doi.org/10.1063/1.2965013

View Table of Contents: http://scitation.aip.org/content/aip/journal/rsi/79/10?ver=pdfcov

Published by the AIP Publishing

\section{Articles you may be interested in}

Data acquisition in a high-speed rotating frame for New Mexico Institute of Mining and Technology liquid sodium aw dynamo experiment

Rev. Sci. Instrum. 84, 104501 (2013); 10.1063/1.4825354

Ultra-high speed photomultiplier tubes with nanosecond gating for fusion diagnosticsa)

Rev. Sci. Instrum. 83, 10D301 (2012); 10.1063/1.4728313

A High-Speed, Multi-Channel Data Acquisition System

AIP Conf. Proc. 728, 438 (2004); 10.1063/1.1843038

Scanning control and data acquisition interface for a spectroscopy diagnostic

AIP Conf. Proc. 563, 209 (2001); 10.1063/1.1374910

High-speed, high-sensitivity aerosol fluorescence spectrum detection using a 32-anode photomultiplier tube detector

Rev. Sci. Instrum. 72, 1831 (2001); 10.1063/1.1344179

\section{Would AC Hall measurement} benefit your research?

See the graph

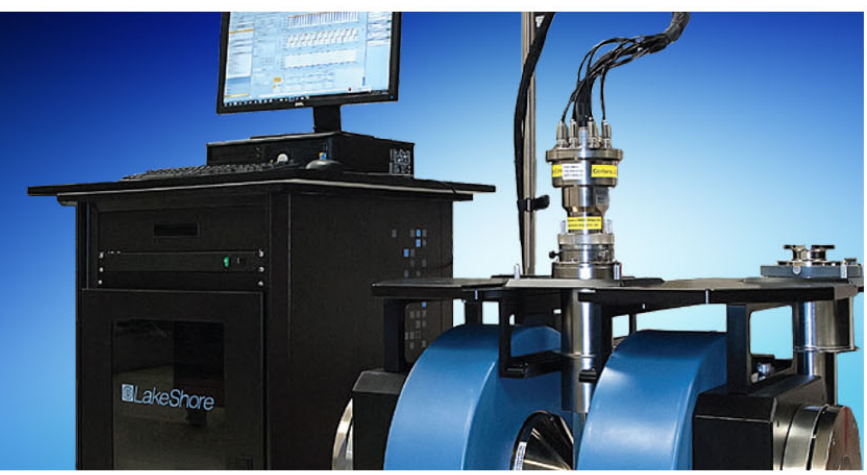




\title{
Imaging photomultiplier array with integrated amplifiers and high-speed USB interface ${ }^{a)}$
}

\author{
M. Blacksell, J. Wach, D. Anderson, J. Howard, S. M. Collis, B. D. Blackwell, \\ D. Andruczyk, ${ }^{\text {b) }}$ and B. W. James ${ }^{\text {c) }}$ \\ Plasma Research Laboratory, Australian National University, Canberra, Australian Capital \\ Territory 0200, Australia
}

(Presented 15 May 2008; received 12 May 2008; accepted 7 July 2008; published online 31 October 2008)

\begin{abstract}
Multianode photomultiplier tube (PMT) arrays are finding application as convenient high-speed light sensitive devices for plasma imaging. This paper describes the development of a USB-based "plug-n-play" 16-channel PMT camera with 16 bits simultaneous acquisition of 16 signal channels at rates up to $2 \mathrm{MS} / \mathrm{s}$ per channel. The preamplifiers and digital hardware are packaged in a compact housing which incorporates magnetic shielding, on-board generation of the high-voltage PMT bias, an optical filter mount and slits, and F-mount lens adaptor. Triggering, timing, and acquisition are handled by four field-programmable gate arrays (FPGAs) under instruction from a master FPGA controlled by a computer with a LABVIEW interface. We present technical design details and specifications and illustrate performance with high-speed images obtained on the H-1 heliac at the ANU. (C) 2008 American Institute of Physics. [DOI: 10.1063/1.2965013]
\end{abstract}

\section{INTRODUCTION}

With high-gain, low noise, and subnanosecond response times, photomultiplier arrays ${ }^{1}$ offer many advantages for high-speed plasma optical imaging ${ }^{2,3}$ and in conjunction with an appropriate scintillator, for imaging soft $\mathrm{x}$-ray plasma emission. ${ }^{4}$ Multiple 16-channel arrays have been used for time-resolved Doppler spectroscopy on the $\mathrm{H}-1$ heliac at the Australian National University, ${ }^{5,6}$ and using an $8 \times 8$ multianode detector array for tomographic imaging of plasma fluctuations. ${ }^{7}$ The devices are also used in a wide number of other applications in nuclear science and medicine.

To be successfully deployed, the parallel photomultiplier tube (PMT) signals need to be appropriately amplified and digitally acquired. Initially, for the $\mathrm{H}-1$ systems, stand-alone arrays of $500 \mathrm{kHz}$ bandwidth transimpedance amplifiers were constructed to amplify and buffer the signals prior to high-speed CAMAC-based digitization. To minimize noise, the detector and amplifier arrays were subsequently encapsulated in a cylindrical PMT housing, which also accommodated $\mu$-metal shielding, a slit and filter assembly, and F-mount lens adaptor. A photograph of this earlier arrangement is shown in Fig. 1. These compact integrated systems were successfully deployed for electron temperature profile measurements on $\mathrm{H}-1$ obtained by imaging and subsequently calculating the intensity ratio of selected atomic helium emission lines from a repetitively pulsed, injected supersonic neutral helium beam. ${ }^{8}$ Typical data are shown in Fig. 2 .

Given the utility of these devices for general purpose

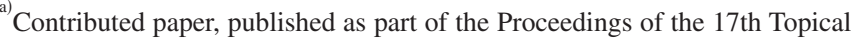
Conference on High-Temperature Plasma Diagnostics, Albuquerque, New Mexico, May 2008.

${ }^{b)}$ Also at Max-Planck-Institut für Plasmaphysik, Greifswald 17491, Germany.

${ }^{c)}$ Also at School of Physics, University of Sydney, New South Wales 2006, Australia.
}

high-speed imaging, we have undertaken to develop a fully integrated, high-speed USB-based "plug-n-play" unit. The 16-channel unit is designed to incorporate necessary optical components, magnetic shielding, an internal high voltage supply for the PMT tube, transimpedance amplifiers, 16 bits, $2 \mathrm{MS} / \mathrm{s}$ data acquisition, on-board memory, and high-speed USB communications interface. The back panel has just four connectors: the USB socket, power connector and external trigger, and acquisition clock inputs. This article describes the electronic layout and mechanical construction of the compact 16-channel camera that is being developed for highspeed optical spectroscopy on the H-1 heliac at the ANU.

\section{HARDWARE OVERVIEW}

The mechanical layout for the PMT camera is shown in Fig. 3. The system accommodates a linear 16-channel Hamamatsu PMT array. The electronics, optics, detector, and shielding are compactly mounted within a $75 \mathrm{~mm}$ diameter aluminum tube with length of $210 \mathrm{~mm}$ which is usually supported on a standard optical mounting rail. A spigot on the front plate accommodates a Nikon F-mount lens adaptor for imaging the external scene. The PMT array and socket are mounted on an internal rail that allows the distance between

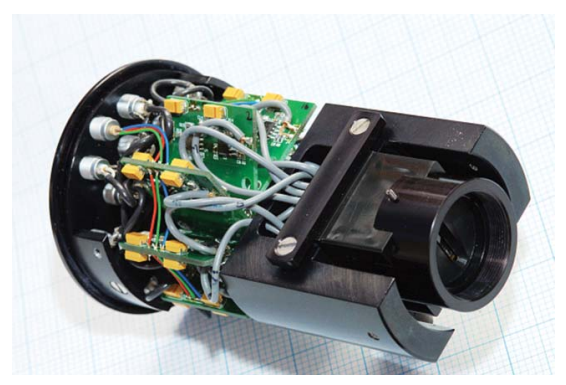

FIG. 1. (Color online) Photograph of the first version of the integrated PMT and amplifier array. 


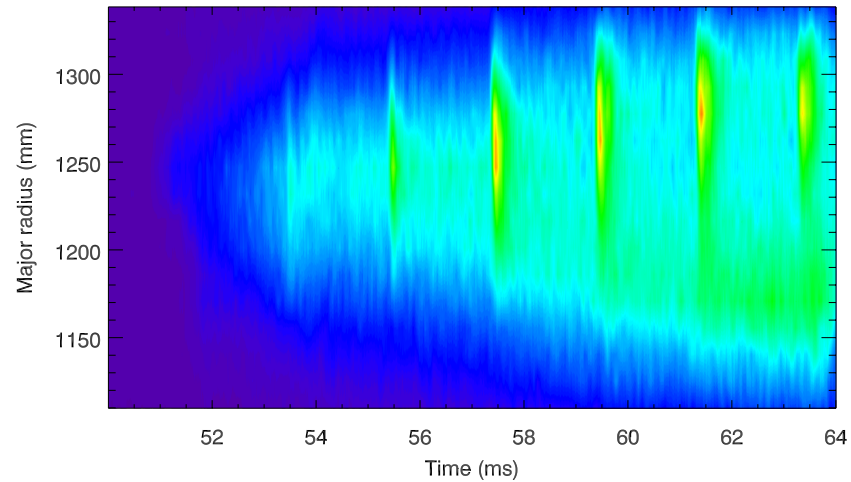

FIG. 2. (Color online) 16-channel light emission data at $504 \mathrm{~nm}$ during multipulse supersonic helium beam injection into an electron-cyclotron heated $\mathrm{H}-\mathrm{He}$ discharge at $0.5 \mathrm{~T}$ in the $\mathrm{H}-1$ heliac.

the camera lens and detector to be adjusted for optimum focusing. The detector is enclosed by a close fitting $\mu$-metal jacket which helps minimize sensitivity to external magnetic fields. Because the sensitive area for each of the 16 detecting surfaces is $\sim 30 \times 1 \mathrm{~mm}^{2}$, provision has been made for the insertion of slit apertures in close proximity to the detector array front surface in order to limit the field of view. In front of the slit is an enclosure to accommodate standard $1 \mathrm{in}$. color filters.

\section{ELECTRONICS}

The electronics consists of sixteen transimpedance amplifiers, 16 analog to digital converters (ADCs), four field programmable gate array (FPGA) devices to control the four ADC boards (four ADCs per board), a master FPGA to process commands and transfer data, and a USB transceiver. The front-end amplifier module and back-end digital hardware unit are connected via an interface card that acts as a break point allowing easy interchange of analog preamplifier units in case it is required to change gain or bandwidth. A block diagram of the circuitry is given in Fig. 4.

\section{A. Transimpedance amplifiers}

The transimpedance amplifiers convert and filter the current from the PMT array to a voltage that is sampled by the ADCs. The transimpedance amplifiers use a low noise $(5.4 \mathrm{nV} / \sqrt{\mathrm{Hz}})$, high impedance $(1 \mathrm{G} \Omega)$, wide gain bandwidth $(180 \mathrm{MHz})$ field effect transistor (FET) operational amplifier (THS4601) to maintain a virtual ground and to minimize loss of signal current. The THS4061 output drives a wide gain bandwidth $(300 \mathrm{MHz})$ current feedback operational amplifier (THS3061) to provide the feedback current drive to maintain a flat gain $(\leq 1 \mathrm{~dB})$ ) and to minimize group delay $(\leqslant 50 \mathrm{~ns})$ through the filter passband $(\leqslant 500 \mathrm{kHz})$. The maximum input current specified for this unit is $10 \mu \mathrm{A}$. The gain and bandwidth of the unit can be changed by replacing a resistor and capacitor respectively.

\section{B. Analog to digital converters}

Four ADCs are mounted on a circular board and are controlled by one FPGA. Four such boards and FPGAs are

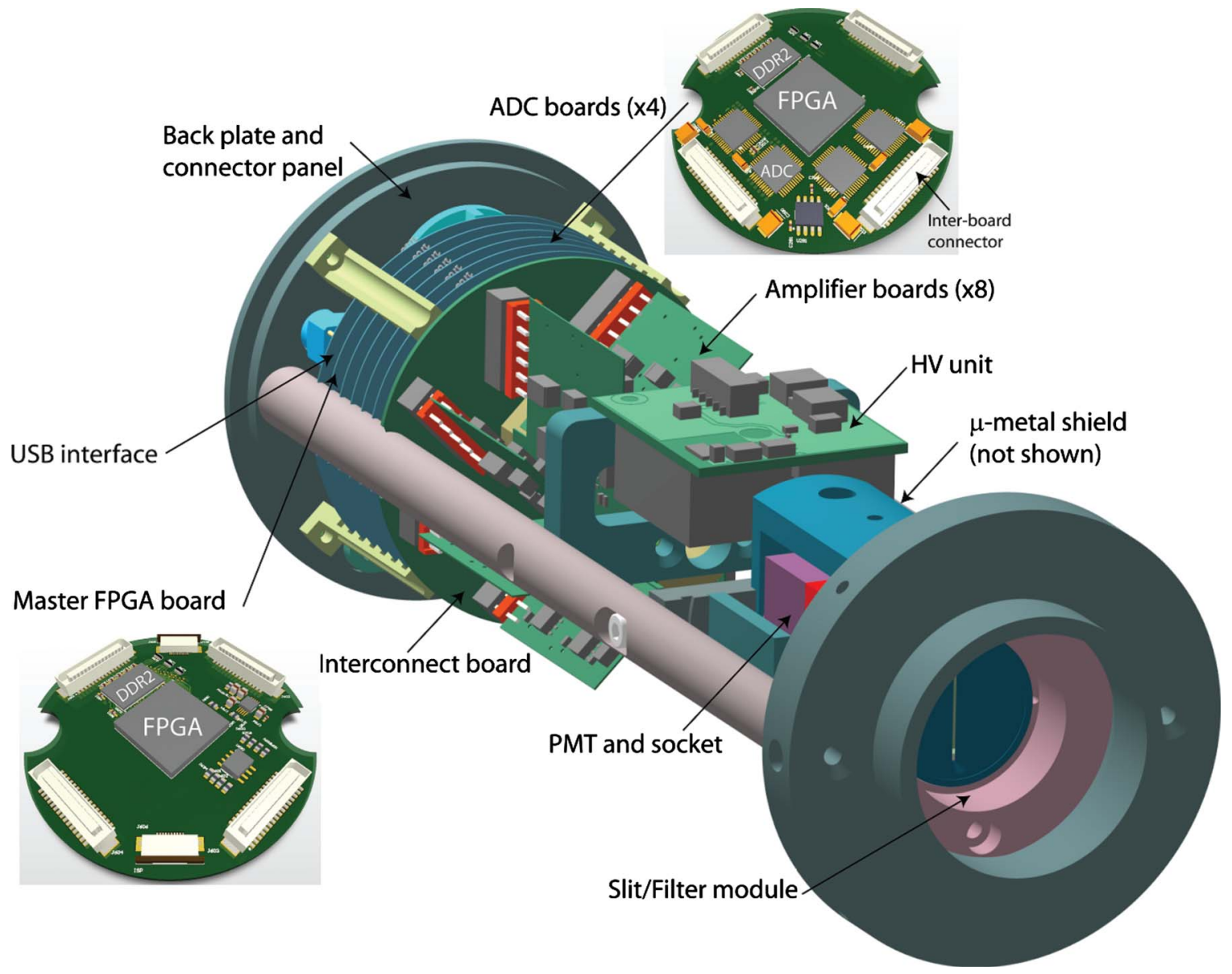

FIG. 3. (Color online) Front view mechanical drawing of the PMT assembly showing ADC and master FPGA boards. 


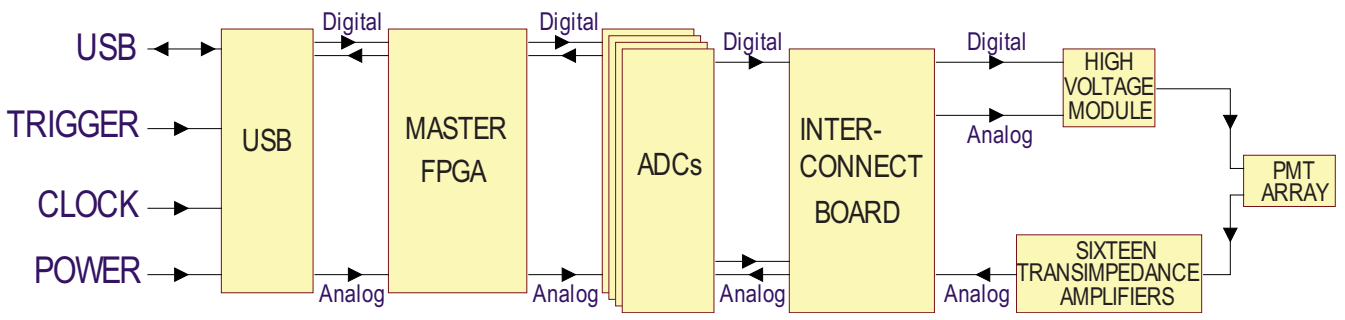

FIG. 4. (Color online) Block diagram of PMT-camera electronics. See text for discussion.

required for acquisition of all 16 channels. The ADCs used are $16 \mathrm{bits}, 2 \mathrm{MS} / \mathrm{s}$, successive approximation register with parallel interface (ADS8411). An ultralow noise reference $(1.8 \mu \mathrm{V}$ voltage noise $0.1-10 \mathrm{~Hz}$, ADR444) is used to provide the $4.096 \mathrm{~V}$ reference for the unipolar input of the ADC and is common between all 16 ADCs. Low noise power supplies $(<125 \mu \mathrm{V})$ provide the $5 \mathrm{~V}$ used for the analog section of the ADC's. The power supply uses a ADR444 reference providing an ultralow noise reference for a low noise $(6 \mathrm{nV} / \sqrt{\mathrm{Hz}})$, precision $(<100 \mu \mathrm{V}$ offset $)$ operational amplifier (AD8620) that drives the gate of a series pass regulator FET. A similar power supply provides the transimpedance amplifiers with a low noise bipolar $( \pm 6.6 \mathrm{~V})$ power supply.

\section{Analog to digital converter FPGA controller}

The FPGA (Altera Cyclone 2 EP2C20F256C6) simultaneously reads the four 16 bit outputs from the ADCs and writes them to the DDR2 memory (Micron MT47H64M16HR). The memory has 1 Gbit capacity and can store up to $\sim 7.8 \mathrm{~s}$ of data at the maximum sampling rate of the ADC's. The memory operates at a clock rate of $166 \mathrm{MHz}$ and is a $4 n$ bit architecture ideal for completing memory transfers so as to not impede the maximum sampling rate of the ADCs. To further minimize noise in the ADC during the crucial conversion stage the DDR2 memory clock circuitry can be disabled thereby removing exposure of the ADC to fast switching digital edges.

\section{Master FPGA controller/USB/HV module}

The master FPGA (EP2C20F256C6) is responsible for managing data transfer from the four ADC FPGAs and for handling commands from the USB 2.0 transceiver (CY7C68001). The various clock, trigger, and data acquisition configurations are established over the USB interface under software control.

The sample clock can be either generated internally or user supplied for synchronization with an external clock source. If generated internally the master FPGA will clock the ADC's at the rate set by software. Both "start" and "stop" trigger modes are supported. In start trigger mode a preset number of samples is acquired following either an externally supplied or internally USB generated trigger. In stop trigger mode, the unit will continuously acquire samples until an external stop trigger is received, whereupon a predefined number of pre- and post-trigger samples are stored.

The master FPGA sets the stable PMT high voltage supplied by a $1 \mathrm{kV}$ source (EMCO C10, Ripple p-p ; $50 \mathrm{mV}$ ) by programming an 8 bit digital to analog converter (DAC) (AD5601). The control voltage from the DAC sets an output resolution of $\sim 4 \mathrm{~V}$ for the $1 \mathrm{kV}$ source. Reset circuitry (LTC1326-2.5) on the USB board monitors power supplies for failure conditions during operation and protects the FPGAs during power up. After power supplies have stabilized at their operating voltage, all FPGA's are released from their reset condition to allow programming and operation.

For improved data integrity and to minimize board space and costs, we have employed DDR2 memory, which features internal data-line termination resistors(on-die termination), to store the data transferred from the FPGA. A reference regulator (TPS51100) on the FPGA boards supplies voltages for the DDR2 termination pull-up resistors and also supplies a stable reference voltage for the memory devices.

The master FPGA board contains the serial configuration device (EPCS16) which is controlled by the master FPGA. After the master FPGA has been programed the four ADC FPGAs are programed too. This serial configuration scheme allows for one serial configuration device and one in-circuit programming connector to program new code for all FPGAs. The software to control the unit has been written in LABVIEW and can be compiled into dynamic linked libraries for use with other languages. The LABVIEW program can be extended to allow post-data-processing.

\section{E. Future developments}

Further plans to modify the unit include a high-speed interface from ADC FPGAs to the master FPGA to allow streaming of data to the PC during acquisition. This will allow data record length to be extended beyond that permitted by the on-board memory device. The unit will also be modified to include a transmission control protocol/internet protocol board which can replace the USB board. With available FPGA resources real time data processing, demodulation and compression of data can be performed.

\footnotetext{
${ }^{1}$ See http://sales.hamamatsu.com/multianode for information about multichannel photo-tube arrays.

${ }^{2}$ R. G. O’Neill, R. J. Smith, C. Akcay, W. T. Hamp, R. Z. Aboul Hosn, T. R. Jarboe, A. J. Redd, P. E. Sieck, G. L. Sutphin, J. S. Wrobel, and HIT-SI Team, J. Fusion Energy 66, 131 (2007).

${ }^{3}$ N. Asakura, M. Takechi, N. Oyama, and T. Nakano, J. Nucl. Mater. 337339, 712 (2005).

${ }^{4}$ L. Delgado-Aparicio, D. Stutman, K. Tritz, M. Finkenthal, R. Kaita, L. Roquemore, D. Johnson, and R. Majeski, Rev. Sci. Instrum. 75, 4020 (2004).

${ }^{5}$ J. Howard, C. Michael, F. Glass, and A. Cheetham, Rev. Sci. Instrum. 72, 888 (2001).

${ }^{6}$ C. Michael, J. Howard, and B. D. Blackwell, Rev. Sci. Instrum. 72, 1034 (2001).

${ }^{7}$ F. Glass, J. Howard, and B. Blackwell, IEEE Trans. Plasma Sci. 33, 472 (2005).

${ }^{8}$ D. Andruczyk, S. Namba, B. W. James, K. Takiyama, and T. Oda, Plasma Devices Oper. 14, 81 (2006).
} 